\title{
A Flocking Algorithm for Second-Order Multi-Agent Systems
}

\author{
Yang $\mathrm{Li}^{1,2}$, Jin Yuan ${ }^{1,2}$, Xuemei Liu ${ }^{1,2}$ \\ 1. Department of Mechanical and Electronic Engineering \\ Shandong Agricultural University \\ Tai'an, China \\ mtlyab@sdau.edu.cn, jyuan@sdau.edu.cn, 1xmywj@126.com \\ 2. Shandong Provincial Key Laboratory of Horticultural Machinery and Equipment \\ Tai'an, China
}

\begin{abstract}
An effective flocking algorithm for multi-agent systems is presented. A type potential function is introduced for avoiding agents' inner collisions, and makes them formulate an lattice pattern. The potential function creates a virtual force is only effective when the distance between two agents is beyond a safe region. With the presented flocking algorithm, agents match the same speed, and keep a certain distance from each other. Simulation results verify the effectiveness of the proposed algorithm.
\end{abstract}

Keywords-multi-agent system; flocking algorithm; potential function

\section{INTRODUCTION}

Multi-agent navigation is a field that has gained increasing attention both in the robotics and the control communities, due to the need for autonomous control of more than one mobile robotic agent in the same workspace. The main feature of formation control is the cooperative nature of the equilibrium of the system. Agents must converge to a desired configuration encoded by the inter agent relative positions. Among the various specifications that the control design aims to impose on the multi-agent team, formation convergence and achievement of flocking behavior are two objectives that have been pursued extensively in the last few years.

Flocking is a phenomenon in which a number of agents move together and interact with each other [1-3]. Flocking control for multiple mobile agents has been studied in recent years [5-8], and it is designed based on three basic flocking rules proposed by Reynolds [6]: flock centering, collision avoidance, and velocity matching. Flocking behavior involves convergence of the velocity vectors and orientations of the agents to a common value at steady state $[9,10]$.

In this paper, the distributed flocking problem for multiagent systems is studied, and a potential function is proposed. In the algorithm we suppose that each agent has interactions with its mates within a desired bounded workspace. The main contributions in this paper include proposed flocking algorithm and potential function.

The rest of this paper is organized as follows. In the next section we present the preliminaries of flocking control and problem formulation. Section 3 describes the design of potential function. Section 4 proposed an effective flocking algorithm. Section 5 shows the simulation results. Finally, Section 6 concludes this paper.

\section{PRoblem Statement}

In [6], it is proposed one kind Geometry of flocking behavior for multi-agent system. A dynamic system with $n$ followers and a virtual leader operating in the same Euclidean space is considered. Let $\left(q_{i}, p_{i}\right) \in R^{2}$ denote the position and velocity of agent $i \in\{1,2, \ldots, n\}$, respectively. In terms of inter-agent distances $d_{i j}$, system geometric object can be described as solutions of the following set of algebraic constraints:

$$
d=d_{i j}=\left\|q_{j}-q_{i}\right\|,(i, j=1,2,3 \ldots, n)
$$

We assume that the motions of the followers are dependent on other neighbors. For the range constraints of sensors, agents cannot always know the information with respect to all other agents. It is known that during the movement of agents, the relative distance between them may change; hence the neighbors of each agent also change. Therefore, we can define a neighborhood set of agent $i$ as follows:

$$
N_{i}=\left\{j \hat{\mathrm{I}} v:\left\|q_{j}-q_{i}\right\|<r\right\}
$$

where $r$ is the maximal range (active range) of the interaction between two agents, and $\|\rtimes\|$ is Euclidean distance.

Considering the interactions between agents are mutual. Therefore, the interrelationships between them can be conveniently described by an undirected graph to model the interaction topology among agents, which consists of a vertex set

$$
V(G)=\left\{v_{i}: i \hat{\mathrm{I}} l_{n}\right\}
$$

and an edge set 


$$
\mathrm{e}(G) \grave{\mathrm{I}}\left\{<v_{i}, v_{j}>: v_{i}, v_{j} \hat{\mathrm{I}} V(G)\right\}
$$

The adjacency matrix $A_{q}=\left[a_{i j}(q)\right] \in R^{n \times n}$ of the undirected graph is a matrix with elements satisfying the properties that if $\left\langle v_{i}, v_{j}\right\rangle \mathrm{I} \mathrm{e}, a_{i j}(q)>0$; otherwise $a_{i j}=0$.

If the adjacent agents can maintain a fixed distance, we find it convenient to define these solutions as lattice-type objects.

Definition 1 ( $\alpha$-Lattice) ${ }^{[6]}$ : An $\alpha$-lattice is a configuration satisfying the set of constraints $r$. And $k=r / d$ as the scale and ratio of the lattice, respectively. Figure 1 shows an $\alpha$-lattice like pattern.

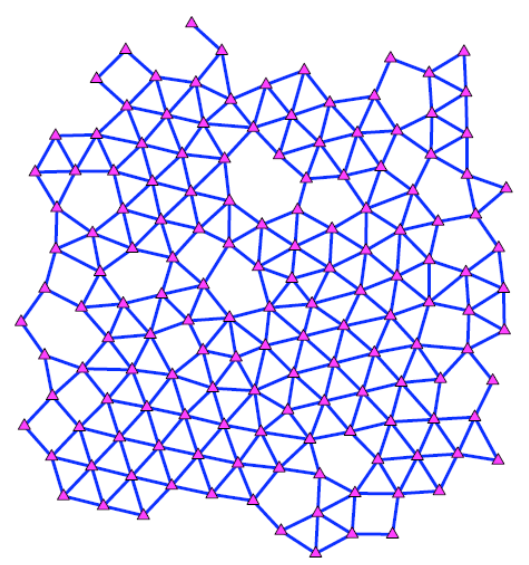

Figure 1: An $\alpha$-lattice

Furthermore, define $u_{i}$ is the control input of agent $i$, in other words, $u_{i}$ can also be regarded as the input command of agent $i$. Then kinematic model of each follower is described by the following equation:

$$
\left\{\begin{array}{l}
\dot{q}_{i}=p_{i} \\
\dot{p}_{i}=u_{i}, \quad i=1,2, \ldots, n .
\end{array}\right.
$$

The flocking control law in [6] controls all agents to form an $\alpha$-lattice configuration. In the system, a virtual force used to align agents to stay close to nearby flock mates, and the different ones will separate from each other. The virtual force component $f_{i}^{\alpha}$, consists of a gradient-based component, is used to regulate the potentials (repulsive or attractive forces),

$$
f_{i}^{\alpha}=\sum_{j \in N_{i}} \phi_{z}\left(q_{j}-q_{i}\right) n_{i j}
$$

where $n_{i j}$ is vector from $i$ to $j$, and $\phi_{z}(\square)$ is positive defined potential function.

\section{Potential Function DesigN}

Each agent can only have interactions with those agents located within a cyclic neighborhood with in specific radius $r$. In this section, we introduce artificial potential field, and define the virtual force associated with the distance. A potential function is a function about adjacent agents' distances and convergence at a certain preset distance. It is also a bounded function. Its value gets larger accompany with the neighbor agents getting too far or too close. The virtual force function could be in different forms, it is designed as following principles:

- Potential function is vector function of agents' distances. The total force is the vector sum of its neighbors'.

- The functions perform repulsion when the distance between agents gets shorter. Otherwise, they perform collection when the distance between two agents gets longer.

- The potential function has a balance distance point $r_{b}$, and has safe distance threshold $\delta$. That means the virtual force between two agents is zero when the their distance falls into the range $\left(r_{b}-\delta, r_{b}+\delta\right)$.

Based on the above principles, its mathematical expression is written as:

$$
\phi_{z i}\left(r_{j i}\right)=\left\{\begin{array}{l}
\sum_{v_{j} \in N_{i}}-\left(\frac{2 k_{r}}{r_{j i}^{2}}+k_{\delta}\right) \boldsymbol{n}_{j i}, \quad 0<r_{j i}<r_{b}-\delta \\
0, \quad r_{b}-\delta \leq r_{j i} \leq r_{b}+\delta \\
\sum_{v_{j} \in N_{i}}\left(\frac{2 k_{r}}{\left(r_{d}-r_{j i}\right)^{2}}+k_{\delta}\right) \boldsymbol{n}_{j i}, r_{j i}>r_{b}+\delta
\end{array}\right.
$$

where $\phi_{z i}$ is the sum of virtual force between $i$ th agent and its neighbors, $r_{j i}$ represents the distance between agent $j$ and $i$, which equals to $\left\|q_{j}-q_{i}\right\|, k_{r}$ and $k_{\delta}$ denote the adjustment parameter of potential function, and $\boldsymbol{n}_{j i}$ is the vector along the line connecting $q_{j}$ to $q_{i}$, which can be calculated as:

$$
n_{j i}=\frac{\left(q_{j}-q_{i}\right)}{\sqrt{1+\eta\left\|q_{j}-q_{i}\right\|^{2}}}
$$

where $\eta$ is an adjustment parameter. 


\section{FLOCKING ALGORITHM}

In this section we present the distributed flocking algorithm in free-space. Agents with the same leader attempt to match velocity with nearby flock mates, but agents with different leaders do not. Now, we write the proposed distributed flocking control law as:

$u_{i}=\sum_{j \in N_{i}} \phi_{z i}\left(r_{i j}\right) n_{i j}-k_{1} \sum_{j \in N_{i}} a_{i j}\left(q_{i}-q_{j}\right)-k_{2} \sum_{j \in N_{i}} a_{i j}\left(p_{i}-p_{j}\right)$

Theorem 1. Consider a system of $n$ mobile agents with dynamics (5). Assume that the initial velocities mismatch and inertias of a group of agents are limited, and system has the initial energy $H_{0}(q(0), p(0))$. The dynamic system (5) under the control input (9), the following statements hold:

- Agents form cohesive flocks moving together, The distance between any agent and its corresponding virtual leader is not larger than $r$;

- Agents form a flocking in a local minimum, and agents with the agents match the same velocity;

- No agent will collide with another.

Proof. The proof procedure is familiar with author's another paper in [11], so it is omitted.

\section{Simulation}

In this section, a simulation example is given to illustrate the theoretical results. The parameters used in this simulation are specified as follows.

Consider the multi-agent system (5) with 50 agents. The initial interactions between them are active as $d_{j i} \leq r$. We choose the range constraints of sensors $r=15$, distance threshold $\delta=4.5$, and $k_{\delta}=1.3$. Figure 2 illustrates the virtual force function we selected.

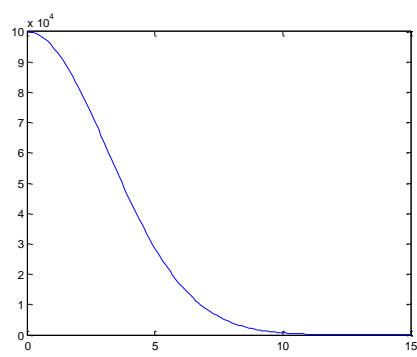

(a)

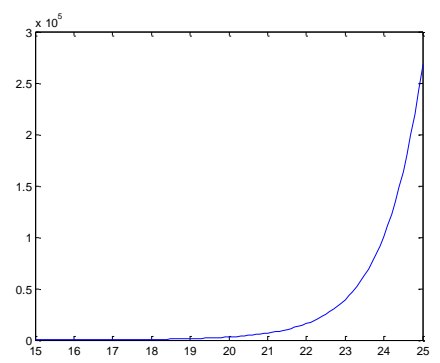

(b)
Figure 2: potential function

The simulation results are shown as Figure 3(a)-(h), all the agents begin with different initial speeds at time $t=0$. Then their speeds converge to the same with the time lapse, and finally reach consensus. Figure 3 (a)-(h) illustrate the 50 agents get $\alpha$-lattice under the flocking protocol. The gents swarm together from each other and hold safe distances between them all the time under the given virtual force.

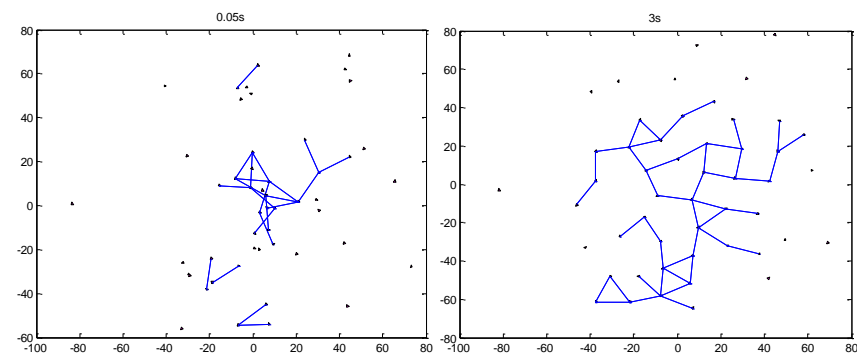

(a)

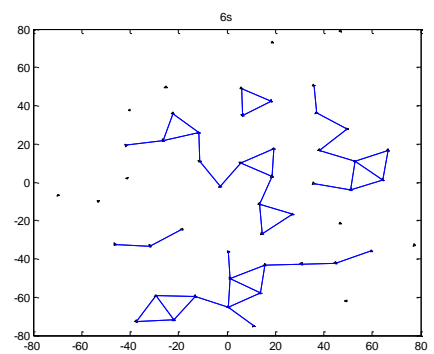

(c)

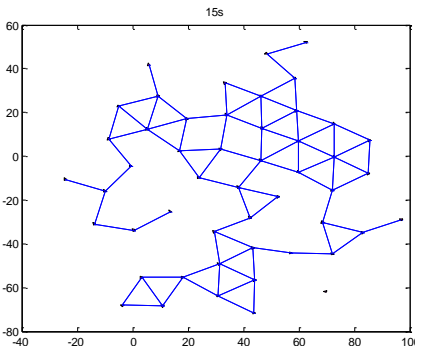

(e)

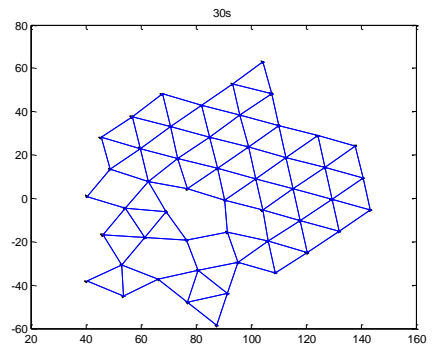

(g) (b)

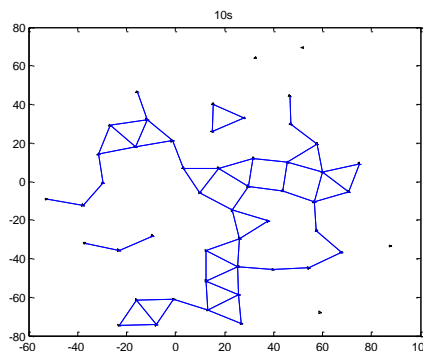

(d)

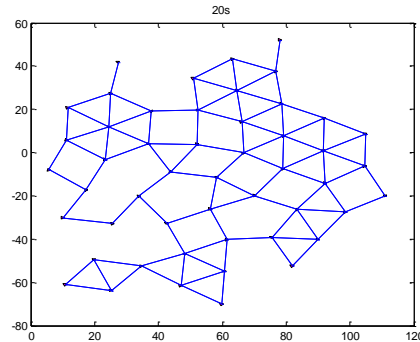

(f)

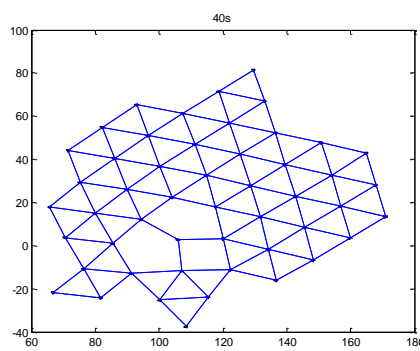

(h)

Figure 3: flocking simulation results

\section{CONCLUSION}

This paper investigates the flocking problem of multi-agent systems led by multi-leader with an undirected topology. Based only on the three classical assumptions for flocking systems, we propose a practical control protocol that pins only a fraction of the agents in the system and allows the agents to follow the leaders when their acceleration is known. Some necessary and sufficient conditions are obtained for the particular case and the paper concludes by applying the theory to some simulated problems and showing their results.

\section{ACKNOWLEDGMENT}

This work supported by the National Natural Science Foundation of China (No. 51475278), Shandong Science and Technology Development Project (No. 2014GGB01483 and 
2013GNC11203), Shandong Provincial Agricultural Equipment Research and Innovation Projects (Grant No. 2015YB201), and the Youth Science and Technology Innovation Fund Of Shandong Agricultural University.

\section{REFERENCES}

[1] Y. Dong, J. Huang, "Flocking with connectivity preservation of multiple double integrator systems subject to external disturbances by a distributed control law". Automatica, Vol. 55(3), pp. 197-203, 2015.

[2] J. N. Jia, L. Wang, "Experimental implementation of distributed flocking algorithm for multiple robotic fish". Control Engineering Practice, Vol. 30(5), pp. 1-11, 2014.

[3] H. Levine, W. J. Rappel, I. Cohen, "Self-organization in systems of selfpropelled particles". Phys. Review. E, Vol. 63, No. 017101, 2000.

[4] H. Su, X. Wang, Z. Lin, "Flocking of multi-agents with a virtual leader". IEEE Transactions on Automatic Control, Vol. 54(2), pp. 293307, 2009.
[5] I. D. Couzin, J. Krause, R. James, "Collective memory and spatial sorting in animal groups". J. Theor.Biol, Vol. 1, pp. 218, 2002.

[6] R. Olfati-Saber, "Flocking for multi-agent dynamic systems: Algorithms and theory". IEEE Transactions on Automatic Control, Vol. 51(3), pp.401-420, 2004.

[7] C. Reynolds, "Flocks, birds, and schools: A distributed behavioral model". Computer Graphics. ACM SIGGRAPH '87 Conference Proceedings, Anaheim, California, Vol. 21(4), pp. 25-34, 1987.

[8] M. C. Fan, H. T. Zhang, M. M. Wang, "Bipartite flocking for multiagent systems". Communications in Nonlinear Science and Numerical Simulation, Vol. 19(9), pp. 3312-3322, 2014.

[9] B. Sharma, J. Vanualailai, U. Chand, "Flocking of Multi-agents in Constrained Environments". European Journal of Pure and Applied Mathematics, Vol. 2, pp. 401-425, 2009.

[10] X. Q. Lu, F. Austin, S. H. Chen, "Flocking in multi-agent systems with active virtual leader and time-varying delays coupling". Commun Nonlinear Sci Numer Simulat, Vol. 16, pp.1014-1026, 2011.

[11] Y. Li, G. Y. Tang, X. X. Yang, "Collision-free consensus in secondorder multi-agent dynamical systems". Control Conference, China, pp. $6245-6249,2012$. 